\title{
High Frequency QRS Analysis From Orthogonal Leads
}

\author{
Josef Halamek $^{1}$, Pavel Leinveber ${ }^{2}$, Marek Malik ${ }^{3}$, Georg Schmidt ${ }^{4}$, Filip Plesinger ${ }^{1}$, \\ Magdalena Matejkova ${ }^{2}$, Jolana Lipoldova ${ }^{2}$, Pavel Jurak ${ }^{1}$ \\ ${ }^{1}$ Institute of Scientific Instruments, CAS, CZ \\ ${ }^{2}$ ICRC, St Anne's University Hospital, Brno, CZ \\ ${ }^{3}$ National Heart and Lung Institute, Imperial College, London, GB \\ ${ }^{4}$ Klinik für Innere Medizin I, Technical University of Munich, Munich, DE
}

\begin{abstract}
We analysed high frequency averaged QRS (HFQRS) in orthogonal leads and different passbands. Three groups of subjects were compared: healthy subjects, ischemic heart disease (IHD) and dilated cardiomyopathy (DCM) patients. Among the IHD group, those with heart failure (HF) symptoms were identified. Investigated parameters included HFQRS maximal amplitude, HFQRS power, and HFQRS fragmentation based on normalized length of the HFQRS line. The study aimed at assessing (1) group differences in relation to the passband, lead, and parameter, and (2) the reproducibility of parameters.

Results: Significant differences were found between healthy subjects and IHD or DCM in all parameters and passbands. Some singularities of significance existed between IHD and DCM. Significant differences were also found between IHD sub-groups with and without HF symptoms, and these existed over more frequency bands.

Conclusion: HFQRS parameters are frequency dependent and this dependency should be tested to eliminate singularities in statistical significances. Differences between groups with or without HF symptoms were found mainly at higher passbands, regardless of deterioration of reproducibility. Lead $X$ appeared to be the lead with maximal differences between groups.
\end{abstract}

\section{Introduction}

High-frequency signal averaged QRS (HFQRS) has been researched for more than 50 years [1] without any analyses of the optimal passband, ECG lead differences, and/or influences of other settings. For the diagnosis of sudden cardiac death risk, late potentials were proposed to be analyzed in a frequency band from $40 \mathrm{~Hz}$ to 250 or $350 \mathrm{~Hz}$ [2], the Reduced Amplitude Zone (RAZ) as a marker of myocardial ischemia was found between 150 and $250 \mathrm{~Hz}$ [3]. The analysis of the time-spatial distribution of electrical activation and corresponding ventricular electrical delay (VED) as the parameter of electrical dyssynchrony $[4,5]$ uses the passband from 150 to $1000 \mathrm{~Hz}$. The higher the frequencies analyzed, the more details are revealed in the distribution of fast action potential changes but also the lower the signal-to-noise ratio is achieved. Most frequently, standard 12-lead ECGs are analyzed while neglecting the orthogonal leads. At the same time, in patients with myocardial ischemia or late potentials, the analysis of orthogonal leads may be sufficient making the analysis simpler. The aim of our study was to assess differences between groups of subjects in relation to passband, lead, and HFQRS parameters. The reproducibility of the parameters was also investigated by comparing independent analyzes of two halves of each available ECG.

\section{Data}

Recordings of 3 groups of subjects were available for the study: Healthy subjects $(\mathrm{N}=182)$, Ischemic Heart Disease (IHD) $(\mathrm{N}=237)$, and Dilated Cardiomyopathy (DCM) patients $(\mathrm{N}=87)$. In the IHD group, 184 patients showed signs of HF symptoms while the remaining 53 patients were free of such symptoms. All ECGs were recorded at St Anne's University Hospital Brno, in a supine position, with high-frequency range $(5 \mathrm{kHz}$ sampling frequency) and high dynamic range (24 bits) ECG system. Lengths of the recordings ranged from 5 to $10 \mathrm{~min}$. Each measurement was separated into two parts of the same length and each part was analyzed independently to test the reproducibility.

Orthogonal recordings of the ART study $[6,7](\mathrm{N}=$ 939 recordings, all sampled at $1600 \mathrm{~Hz}$ ) were also available. These were separated into two halves (each 15 min long). The processing of ART study recordings was blinded, without any access to the corresponding clinical data and diagnoses. As previously published, of the 939 ART patients, 72 suffered from all-cause mortality (ACM), 38 from cardiac death (CM), and 14 from sudden cardiac death (SCD) during a nominal follow-up of 5 
years.

\section{Analysis}

At the first step, QRS complexes were detected [8] and sorted [9]. Amplitude envelopes in 12 frequency windows (bandwidth $100 \mathrm{~Hz}$, middle frequency f0 from 100 to 650 $\mathrm{Hz}$, in steps of $50 \mathrm{~Hz}$ ) were computed and regular (sinus beats) HFQRS complexes were averaged. Analyzed parameters were HFQRS maximal amplitude (Amax), HFQRS power (PW) and HFQRS fragmentation (FR) based on normalized length of HFQRS line. Instead of the potentially problematic binary (yes/no) definition of fragmentation, we introduced a continuous variable based on the normalized QRS length, defined as:

$\mathrm{FR}=\mathrm{QRSL} /($ Amax-Amin $) / 2-1$

where QRSL is the length of HFQRS line, and Amax and Amin are maximal and minimal HFQRS amplitudes, respectively. Intra-subject relative differences were defined as:

DIFrel $=\left|\mathrm{m}_{1}-\mathrm{m}_{2}\right| /\left(\mathrm{m}_{1}+\mathrm{m}_{2}\right) / 2 * 100$

where $\mathrm{m}_{\mathrm{i}}$ is the parameter in the $\mathrm{i}$-th half of measurement.

\section{Results}

Frequency dependency of Amax, PW, and FR are shown in Fig. 1 together with the reproducibility defined as the relative differences between two halves of individual recordings. The mean levels over groups of subjects are presented. Single leads X, Y, Z are presented at Amax, PW, and FR. Mean levels over leads are used for DIFrel, as the reproducibility predominantly depends on the parameter and analyzed passband whilst the levels of reproducibility over leads are similar. Statistically significant differences existed between healthy and IHD or DCM in all parameters, leads, and passbands. Comparing IHD and DCM, statistically significant differences existed in FR in some passbands, mainly in the leads $X$ and $Z$ (Fig. 2a). Statistically significant differences also existed between subgroups of IHD patients with and without HF symptoms, as shown in Fig. $2 \mathrm{~b}$, c, and d.

Blinded analysis of the ART study data was performed for one passband with $\mathrm{f} 0=250 \mathrm{~Hz}$. The corresponding reproducibility of data is shown in Fig. 1. The initial analysis with $\mathrm{f} 0=250 \mathrm{~Hz}$ suggested differences between the groups of survivors and groups of patients with outcome events (ACM, CM and SCD) in FR measured in lead X. Nevertheless, subsequent analyzes with different passbands (Fig. 3) did not reproduce these statistically significant differences.
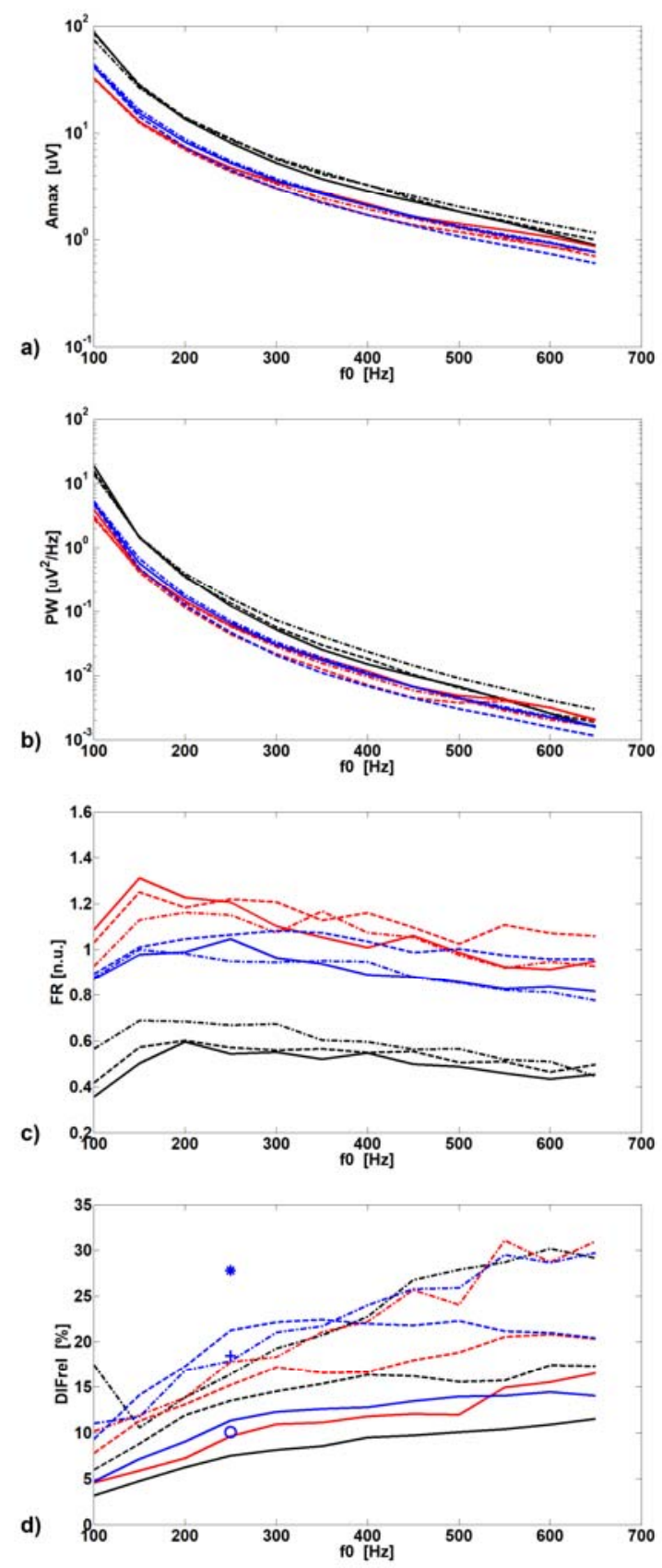

Fig 1. Frequency dependence of mean parameter levels in subject groups. Healthy subjects, IHD, and DCM patients are shown in black, blue, and red respectively. For Amax, $\mathrm{PW}$, and FR, leads $\mathrm{X}, \mathrm{Y}$, and $\mathrm{Z}$ are shown in solid, dashed and dashed-dotted lines, respectively. For DIFrel, mean levels over leads are shown. Reproducibility of Amax, PW, and FR is represented by solid, dashed and dasheddotted lines, respectively. In the ART study data, the blue symbols 'o','+', and '*' mark the reproducibility of

Amax, PW, and FR, respectively. 

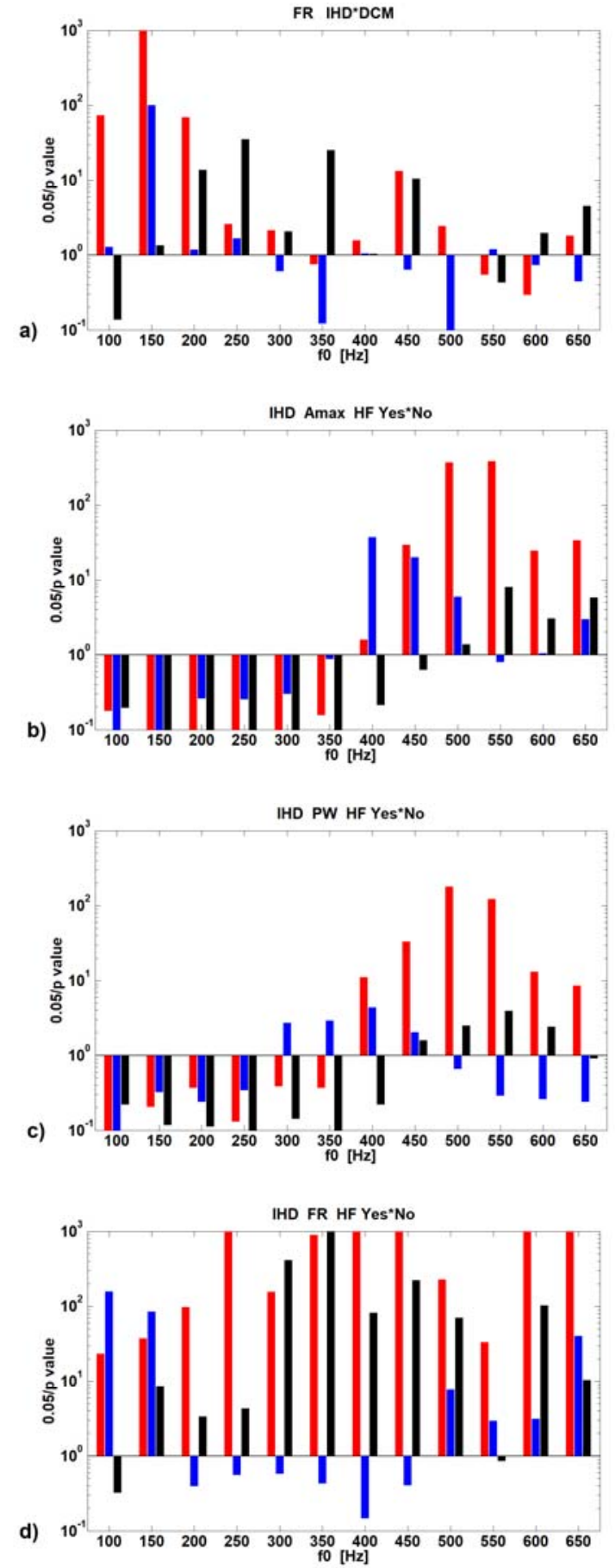

Fig. 2. Statistical significances of differences between groups. Leads X, Y, Z are shown in red, blue, and black, respectively.

\section{Discussion}

Amax and PW decreased logarithmically with f0 whilst their DIFrel increased. The best reproducibility was observed in healthy subjects for Amax (Fig. 1a).
Comparisons of the reproducibility of Amax and PW in the Brno and ART data (Fig. 1d, note that ART approximately corresponds to the IHD group), the reproducibility in the ART study data was marginally better (Amax) or similar (PW). Analyzed ART data were longer (30 min) and possible fluctuation in data given by breathing and other cardiovascular control systems is minimized by averaging.

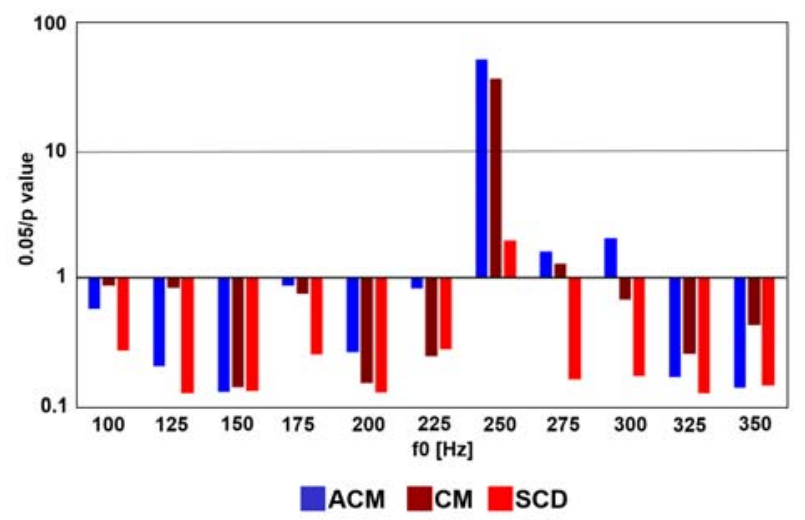

Fig. 3. Statistical significances of differences in FR in lead X between subgroups of ART study and the survivors of the ART study population.

We used the normalized length of the QRS line as the parameter that describes QRS fragmentation. This is a simple definition allowing expressing the fragmentation on a continuous scale. Nevertheless, signal preprocessing is needed and reasonable signal-to-noise ratio is required. Significant differences existed between groups of subjects in FR level. FR had the maximal level at f0 around 200 $\mathrm{Hz}$. With increasing or decreasing f0, the FR level decreased. The reproducibility of FR was poorer compared to that of Amax or PW. Comparing FR reproducibility between Brno data and ART study, the FR reproducibility in the ART study was significantly worse. The lower dynamic range of the ECG system used in ART study and the lower signal-to-noise ratio in ART recordings might have contributed to this difference.

Statistically significant differences existed in all parameters and frequency bands between healthy subjects and IHD or DCM groups. Some singularities of statistical significance existed also between IHD and DCM groups (e.g. the FR parameter shown in Fig. 2a). Such singularities demonstrate that the analysis of differences based on the fixed frequency band and neglecting the frequency dependency of HFQRS may be misleading. Presented parameters did not truly differentiate between the IHD and DCM group. This might have been caused by the inhomogeneity of the groups. On the contrary, there were significant differences between HF and nonHF subgroups of IHD patients (Fig. 2b, c, d). These were found over more passbands with stronger differences at higher frequency bands. Higher frequency components 
better describe the time-spatial distribution of action potential phase 0 . This analytical benefit overwhelms the deterioration of reproducibility. Not surprisingly, the parameter FR showed the strongest results (mainly in lead $\mathrm{X})$.

The fragmented QRS complex is known to be associated with increased mortality and arrhythmic events $[10,11]$. Our analysis was based on orthogonal leads and used a simple FR definition that eliminated subjective decisions. Generally, the HFQRS analysis is promising, simple and easy to perform. Nevertheless, our results need to be confirmed within multiple independent data collections. It is important to collect high-quality ECG signals much longer compared to the standard 10-second recordings. The clinical community involved in future studies should consider the potential value of high-quality signal collection [12].

The limitations of our study include the lack of detailed diagnoses and long-term follow relevant to the Brno recordings. The blinded analysis that was applied to the ART study data involved statistical analysis performed in London but only a limited number of parameters were tested. Only FR in lead X was tested over more frequency bands.

\section{Conclusion}

The reproducibility and possible diagnostic contribution of HFQRS parameters were tested. HFQRS properties are frequency dependent and this dependency should be considered in any future analysis. Using a fixed frequency band for the HFQRS analysis without appropriate justification and/or verification can be substantially misleading. Statistically significant differences between groups were observed mainly at higher frequencies and were not hampered by lower reproducibility. The lead $\mathrm{X}$ signals appeared to provide maximal differences between compared groups.

\section{Acknowledgements}

The research was supported by Czech Science Foundation, project GA17-13830S, by Ministry of Education, Youth and Sports of the Czech Republic (project LO1212 and LQ1605) and by the Czech Academy of Sciences (project RVO:68081731).

\section{References}

[1] Goldberger AL, Bhargava V, Froelicher V, Covell J. Effect of myocardial infarction on high-frequency QRS potentials. Circulation 1981; 64:34-42.

[2] Breithardt G, Cain M, El-Sherif N, Flowers N. et al. Standards for analysis of ventricular late potentials using high-resolution or signal-averaged electrocardiography. JACC 1991; 17: 999-1006.
[3] Amit G, Granot Y, Abboud S. Quantifying QRS changes during myocardial ischemia: Insights from high frequency electrocardiography. J Electrocardiol. 2014; 47 (4):505-11.

[4] Jurak P, Halamek J, Meluzin J, Plesinger $F$ et al. Ventricular dyssynchrony assessment using ultra-highfrequency ECG technique. J Intervent Cardiac Electrophysiol. 2017; 49: 245-54.

[5] Plesinger F, Jurak P, Halamek J, Nejedly $P$ et al Ventricular electrical delay measured from body surface ECGs is associated with cardiac resynchronization therapy response in left bundle branch block patients from the MADIT-CRT trial (Multicenter Automatic Defibrillator Implantation-Cardiac Resynchronization Therapy). Circ Arrhythm Electrophysiol 2018; 11 (5): e005719. DOI: 10.1161/CIRCEP.117.005719;1-12.

[6] Barthel P, Bauer A, Müller A, Huster KM, Kanters JK, Paruchuri V, Yang X, Ulm K, Malik M, Schmidt G. Spontaneous baroreflex sensitivity: Prospective validation trial of a novel technique in survivors of acute myocardial infarction. Heart Rhythm 2012; 9:1288-94.

[7] Schmidt G, Malik M, Barthel P, Schneider R et al. Heartrate turbulence after ventricular premature beats as a predictor of mortality after acute myocardial infarction. Lancet. 1999; 353(9162):1390-6.

[8] Plesinger F, Jurco J, Jurak P, Halamek J. Robust multichannel QRS detection. Computing in Cardiology. 2014; 41:557-560.

[9] Plesinger F, Jurco J, Halamek J et al. Multichannel QRS Morphology Clustering Data Preprocessing for Ultra-HighFrequency ECG Analysis, Cardiotechnix 2015; pp. 11-19.

[10] Das MK, Suradi H, Maskoun W et al. Fragmented wide QRS on a 12-Lead ECG a sign of myocardial scar and poor prognosis. Circ Arrhythm Electrophysiol. 2008; 1: 258-68

[11] Redfors B, Kosmidou I, Crowley A et al. Prognostic significance of QRS fragmentation and correlation with infarct size in patients with anterior ST-segment elevation myocardial infarction treated with percutaneous coronary intervention: Insights from INFUSE-AMI trial. Int $\mathrm{J}$ Cardiol. 2018; 253: 20-4

[12] Malik M, Buxton A, Huikuri $H$ et al. Noninvasive electrophysiology in risk assessment and screening. Heart Rhythm. 2018; 15: 803-4

Address for correspondence.

Josef Halamek

Institute of Scientific Instruments of the CAS

Kralovopolska 147, 61264 Brno

Czech Republic

josef@isibrno.cz 